\title{
Knowledge Promotes Quality Management: A Case Study of Quality Problem-Solving in Two Automotive Plants
}

\author{
Zhaoguang Xu*, Yanzhong Dang \\ Institute of System Engineering, Dalian University of Technology, Dalian, China \\ Email: ${ }^{\star}$ zhgxu@dlut.edu.cn
}

How to cite this paper: $\mathrm{Xu}, \mathrm{Z}$. G., \& Dang, Y. Z. (2021). Knowledge Promotes Quality Management: A Case Study of Quality Problem-Solving in Two Automotive Plants. American Journal of Industrial and Business Management, 11, 933-953.

https://doi.org/10.4236/ajibm.2021.118057

Received: August 2, 2021

Accepted: August 23, 2021

Published: August 26, 2021

Copyright $\odot 2021$ by author(s) and Scientific Research Publishing Inc. This work is licensed under the Creative Commons Attribution International License (CC BY 4.0).

http://creativecommons.org/licenses/by/4.0/

\begin{abstract}
Quality management is a vital link to ensure product quality in the automobile production process. This paper investigates problem-solving of two automotive plants and explores the factors that influence the quality improvement in the organisation. The case studies analyse the status quo and problems in the quality management organisation, problem-solving process, and team, as well as quality management information system with particular emphasis on how each plant uses data, information, and knowledge to solve quality problems from the perspective of knowledge management. The result shows that there is a lack of utilisation of data, information, and knowledge in problem-solving. Based on the analysis result and the demands of plants for improving the efficiency and effectiveness of problem-solving, we propose a knowledge management based intelligent problem-solving system (IPSS). At the same time, a five-tier environment construction for the successful implementation of IPSS is proposed. The main shortcomings identified are common to many other plants and companies worldwide. The suggestions and proposals put forward are of great significance for manufacturing enterprises to improve the efficiency and effectiveness of quality problem-solving.
\end{abstract}

\section{Keywords}

Quality Management, Knowledge Management, Automotive Industry, Problem-Solving, Case Study

\section{Introduction}

With the continuous improvement of living standards, the demands of consumers regarding the quality of automotive products are also increasing (Herrmann, ${ }^{\star}$ Corresponding author. 
Henneberg, \& Landwehr, 2010). In addition to increased competitiveness about product performance, price, and brand, quality is a crucial factor. Various studies show that product quality has a significant relationship with brand image and brand trust (Hanaysha, Hilman, \& Abdul-Ghani, 2014). Problem-solving plays a vital role in quality management to ensure the high-quality product (Liang \& Zhang, 2010). Traditional models of problem-solving assume that a structured process of problem definition, problems analysis, and root causes identification, generation, and selection of solutions, testing, and evaluation of solutions (Macduffie, 1997; Xu \& Dang, 2020), while this process mainly relies on human experience and overlooks the importance of data, as well as organisational knowledge (Linderman, Schroeder, Zaheer, Liedtke, \& Choo, 2004; Berends, 2005). Because of staff mobility and inexperienced new employees, problem-solving is often a time consuming and cost-intensive process, which makes the organisational knowledge play a key role in problem-solving (Postrel, 2002; Peachey \& Hall, 2006).

From the perspective of improving quality management and problem-solving, many scholars recognise the importance of knowledge management (KM) in quality management (Xu, Dang, \& Munro, 2018). Early scholars analysed the relationship between quality and learning. Fine (1986) first created an analysis model that studied failure costs and consistent costs, and found that the best quality level increases as learning time increases. Learning has a strong connection with knowledge creation. Choo, Linderman, and Schroeder (2007) develop a framework for learning and knowledge-based quality improvement by integrating the two perspectives of learning and knowledge creation. Ibrahim and Heng (2013) examined the relationship between learning and knowledge creation and found learning will promote organisation knowledge creation.

With the development of informatisation, the value of massive amounts of data is increasingly valued by people (Mons, Van, Chichester, Hoen, den Dunnen, van, \& Schultes, 2011). Another stream of KM research in quality management focuses on using big data technology to obtain knowledge from quality data since knowledge discovery from data is a trend in the era of the knowledge economy (Ruikar, Anumba, \& Egbu, 2007; Chen, Deng, Wan, Zhang, Vasilakos, \& Rong, 2015). The trend towards profound knowledge in the future of quality management is emphasised by Stanleigh (Stanleigh, 2013). Using data mining and knowledge discovery technology to provide support for quality management is the focus of intelligent quality management (Wang, 2009).

Not only these data mining technologies, but intelligent systems with KM feature are also highly recognised by scholars to improve quality improvement and problem-solving. Lari (2004) discussed an intelligent system regarding the corrective and preventive action (CPA) process which employs both case-based reasoning (CBR) and decision support systems (DSS) components. Ch et al. (2009) proposed a model which contains a new component of KM and introduced an intelligent quality management process model (IQMP). Srikanth et al. (2010) proposed an IQM expert system with a Post Analysis of Actionable Knowledge Discovery (PA-AKD) framework. The application of the intelligent 
system in the more specific area of quality management is also explored. Erginel and Şentürk (2015) developed an intelligent system to eliminate or reduce uncertainty on data of control charts in SPC. With the application of the Exponentially Weighted Moving Average (EWMA), Aslan, Ekinci, and Toy (2016) suggested an intelligent control and repair system such that in a production system, machines are individually controlled and repaired when an out-of-control signal is triggered in the zone with the tight control limits. Liu et al. (2014; 2013a; 2013b; 2012), Asan and Soyer (2016) combined the fuzzy evidential reasoning approach with the belief rule-based (BRB) methodology to identify potential failure modes and their effects (FMEA) with uncertain information. By mining unstructured texts, $\mathrm{Xu}$ et al. (2020) proposed a method to construct the component-failure mode (CF) matrix automatically for FMEA.

Most of these studies dig out knowledge from quality management data from a technical perspective, but lack a management perspective to analyse why the problem-solving process lacks knowledge and how knowledge can promote quality management. Based on the current research, our study below will further promote the research on the combination of quality management and KM. We investigate the quality management, especially problem-solving of two typical automotive plants to explore the key factors affecting problem-solving. Moreover, based on these critical factors, we intend to seek an intelligent approach to increase the efficiency and effectiveness of problem-solving.

This paper is organised as follows: In Section 2, we introduce the case study backgrounds and research questions. Section 3 explores and compares the problem-solving situations in two case plants and the problems they faced during problem-solving. The questionnaire analysis is conducted in Section 4. In Section 5 , we propose the IPSS that will promote the effectiveness and efficiency of problem-solving. The final section concludes the entire paper and presents future work.

\section{Case Study Backgrounds, Methodology and Research Questions}

\subsection{Case Plants Background}

Our research sites for this study were two vehicle manufacturing plants in China. The case plant, BBA, founded in 2003 in Shenyang, China, is a joint venture of BMW and a local Chinese Automotive company. The case company, SGM, founded in 2004 in Shenyang, is a joint venture of General Motors and a local Chinese Automotive company. The two companies, as Sino-foreign joint ventures, own very mature quality management systems. Their quality management processes and systems are introduced from their foreign parent companies. Additional background information for these two plants is presented in Table 1, including plant characteristics not investigated during the field visits, plant productivity and production scale, product category, and quality problems from Initial Quality Survey conducted by J.D. Power. Average quality for the other companies is included for comparison purpose. 
Table 1. Background information on case plants.

\begin{tabular}{cccc}
\hline Characteristic & BBA & SGM & Industry Average \\
\hline Plant Age & Built in 2003 & Built in 2004 & - \\
Product Category (Vehicle Models) & 7 & 3 & - \\
Productivity (Jobs Per Hour) & 64.2 & 60 & 128 \\
Quality* (Problems per 100 vehicles) & 108 & 120 & - \\
Production Scale (Vehicles per day) & $1000-1100$ & $690-720$ & - \\
\hline
\end{tabular}

^Source: J.D. Power 2020 Initial Quality Survey (IQS).

\subsection{Research Methodology and Fieldwork Activities}

This article researches two case companies in the form of interviews, questionnaires and field trips. The researchers stayed five weeks at BBA, three days a week. We talked with 24 interviews and participated in 20 quality-related meetings during the period. We spent four weeks at SGM, and three days each week. At this plant, we interviewed 18 people and participated in 14 quality meetings. In the horizontal view, the interviewees come from related departments such as quality, R \& D, procurement, and production. Vertically speaking, the interviewees cover plant managers, workshop directors, quality managers, quality specialists, engineers, and operators.

In order to better acquire employees' understanding and advice on quality management, especially on problem-solving, we designed an "Automobile Quality Management Questionnaire." The questionnaire contains closed questions and open questions. It mainly consists of quality awareness, quality tools, quality management methods, problem-solving culture, teams, and processes, as well as data, information and knowledge in quality management. The main questions contained in the questionnaire are presented in the Appendix. In BBA and SGM, 256 questionnaires and 189 questionnaires were distributed. The number of questionnaires returned was 228 and 169, of which valid questionnaires were 220 and 165 . The effective recovery rates were $85.9 \%$ and $87.3 \%$, respectively.

At all of these plants, we received permission to walk around the plant, to talk with workers, team leaders, engineers, quality analysts, production and quality managers, and to gather relevant documents. In each plant, we asked about the same questions, observed the same production processes and quality focused group activities.

\subsection{Research Questions}

During the investigation, we mainly considered the factors that affected the problem-solving, including quality management organisational structure, problem-solving process, problem-solving team, as well as the use of data, information, and knowledge in problem-solving. In the interviews and fieldwork activities, we asked the following questions:

1) Problem-solving process 
Is there a structured problem-solving process? Is there a standard description of problems? How to evaluate the severity of the problem? How to conduct root cause analysis? Is the plant equipped with mature technology to analyse problems?

2) Problem-solving team

Who is involved in problem-solving? Is the team leader clear? Are the participants in problem-solving team qualified? How broad is the organisational knowledge utilised in problem-solving?

3) Use of data, information, and knowledge

How to collect and use data, information, and knowledge to solve the problem? Can quality data be recorded and documented with accuracy and shared among departments?

In addition to these questions, we also surveyed the quality organisation structure and quality management information systems. We collect, summarise and analyse the current situation and existing problems of quality management and problem-solving of the two plants. The use of data, information, and knowledge is paid more attention. Combine the results of our analysis with the actual demands of the company, method, and path to improve the efficiency and effectiveness of problem-solving by fully exploiting quality solving data is proposed.

\section{Analysis of Related Factors Affecting Problem-Solving}

In this section, we analyse five aspects that will influence problem-solving efficiency and effectiveness, including quality management organisation structure, quality management information system, problem-solving process, and team, as well as the use of data, information, and knowledge. For each part, we first present the actual situation in the case plants and evaluate the similarities and differences between them.

\subsection{Quality Management Organisation Structure}

Both BBA and SGM have two levels of quality management department, plant level, and workshop level. The plant Total Quality Management (TQM) department at BBA is responsible for the quality management system, product audit, problem-solving, vehicle validation, geometric measurement, fault analysis/diagnostics, laboratories, start-up and change control, and various plant projects. The TQM department at SGM includes Quality Assurance, Vehicle Quality, and Quality Engineering. Each function contains a lower level of quality sub-departments.

In BBA, each workshop has its quality department and the final product audit set in the workshop reports for the workshop. The plant quality makes the final audit from the customer's point of view. However, in SGM, the final audit station in each workshop belongs to the plant quality department. This kind of organisation strategy in BBA is more modern than SGM. While it also means each workshop checks its products, which may hide some quality problems for 
workshop Key Performance Indicator (KPI). Although the main audit of the plant will inspect the vehicles, the later the problem is found, the more money they should pay.

Another big difference between the two plants lies in the analysis centre. BBA's plant quality department has its analysis centre. However, SGM does not have its analysis centre. Analysis centre is quite important especially for quality problems related to suppliers. BBA can analyse the supplier parts in plant analysis centre, while SGM can only rely on the supplier's report. When we talked with quality managers at SGM, they also believe that it is a risk in problem-solving.

\subsection{Quality Management Information System}

Both BBA and SGM have a complete set of the quality management information system; this paper mainly focuses on defect record system and problem management system. These two kinds of systems for each plant are listed in Table 2.

The defect record system (DRS) is used to record the quality defects found in various aspects of the production process, and also track the rework of these defects. For each car, if one of the records in the DRS is red, the car cannot be viewed as a customer car. Although the names of the DRS of BBA and SGM are inconsistent, they have similar functions.

Problem Management System (PMS) is an essential platform for recording related problem-solving data. For BBA, as an IT support tool for Problem Management Process (PMP), Product Quality Management (PQM) has penetrated all business departments and runs through the entire process chain. Since the introduction of PQM in July 2010, one in every 10 BBA employees has used PQM, which has also made PQM a pillar of BBA's product quality management and problem-solving. However, the system is subject to intellectual property filtering. BBA can only use a part of its functions and view a part of data. What's more, this system is opened to some key users.

Problem Communication Report (PCR) system of SGM is also introduced by the United States parent company. Before the introduction of PCR, the company stored the data and critical information in the problem-solving process in word documents. Each problem has a dedicated word document. Since 2008, SGM has been using the PCR system. Due to the intellectual property agreement between the joint venture and their foreign parent companies, SGM also has limited access to the data in the PCR.

A search function is integrated into both PQM and PCR. When a new quality problem occurs, users can search the system by inputting the keyword. This search

Table 2. Typical quality management information systems at BBA and SGM.

\begin{tabular}{ccc}
\hline Plant & BBA & SGM \\
\hline Defect Record System (DRS) & IPS-Q & GSIP \\
Problem Management System (PMS) & PQM & PCR \\
\hline
\end{tabular}


engine-like function provides users with related data and information. However, it is a painful task to pick up useful information from a large number of search results. So problem solvers prefer to consult relevant personnel through emails and telephones to acquire the historical information about the problem. During our interviews with these system users, we found that they urgently need the system to directly and accurately provide relevant knowledge of quality problems.

\subsection{Problem-Solving Process}

The research in this section introduced the status quo of the problem-solving process of the two case companies and compared their similarities and differences.

BBA has a complete quality management process, which is called the Problem Management Process (PMP). As shown in Figure 1, the PMP is divided into 14 stages, each stage corresponds to a different task, and different roles are also involved.

Sensors are employees and systems that detect and indicate any variances from nominal, describe them in a PQM incident and then transfer them to a Melder (Incident Indicator). As a general rule, once the problem processing is complete, they carry out the secondary proof of effectiveness. Melders check, accept and reconcile the incidents that the Sensors have picked up, assign them to existing problems, generate new problems, or open a closed problem that, once assessed, can be moved into the steering level. Q-Lenkers steer and control the PMP process in status $1-100$. The person responsible for the solution, also known as Problem Owner (LV) manages the problems in status 30 - 90, which includes generating immediate measures, carrying out a systematic cause analysis and finding a solution. The person responsible for an individual activity (MAV) is responsible for processing the solutions timely to problems at status $60-70$. His job is to make sure that validation trials can confirm the primary proof of effectiveness so that the cause of the problem is permanently eliminated.

The task in each status is listed in Table 3.

Compared with BBA PMP, SGM problem-solving process is relatively more concise. Figure 2 shows the problem-solving process, the responsible persons at each stage, and the status of the problem.

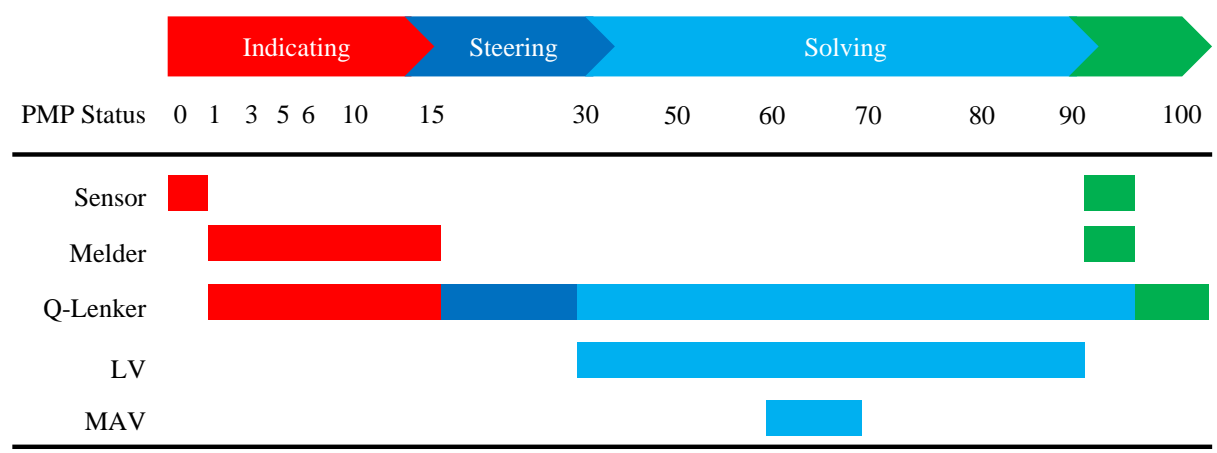

Figure 1. Problem management process at BBA. 
Table 3. Problem management process at BBA.

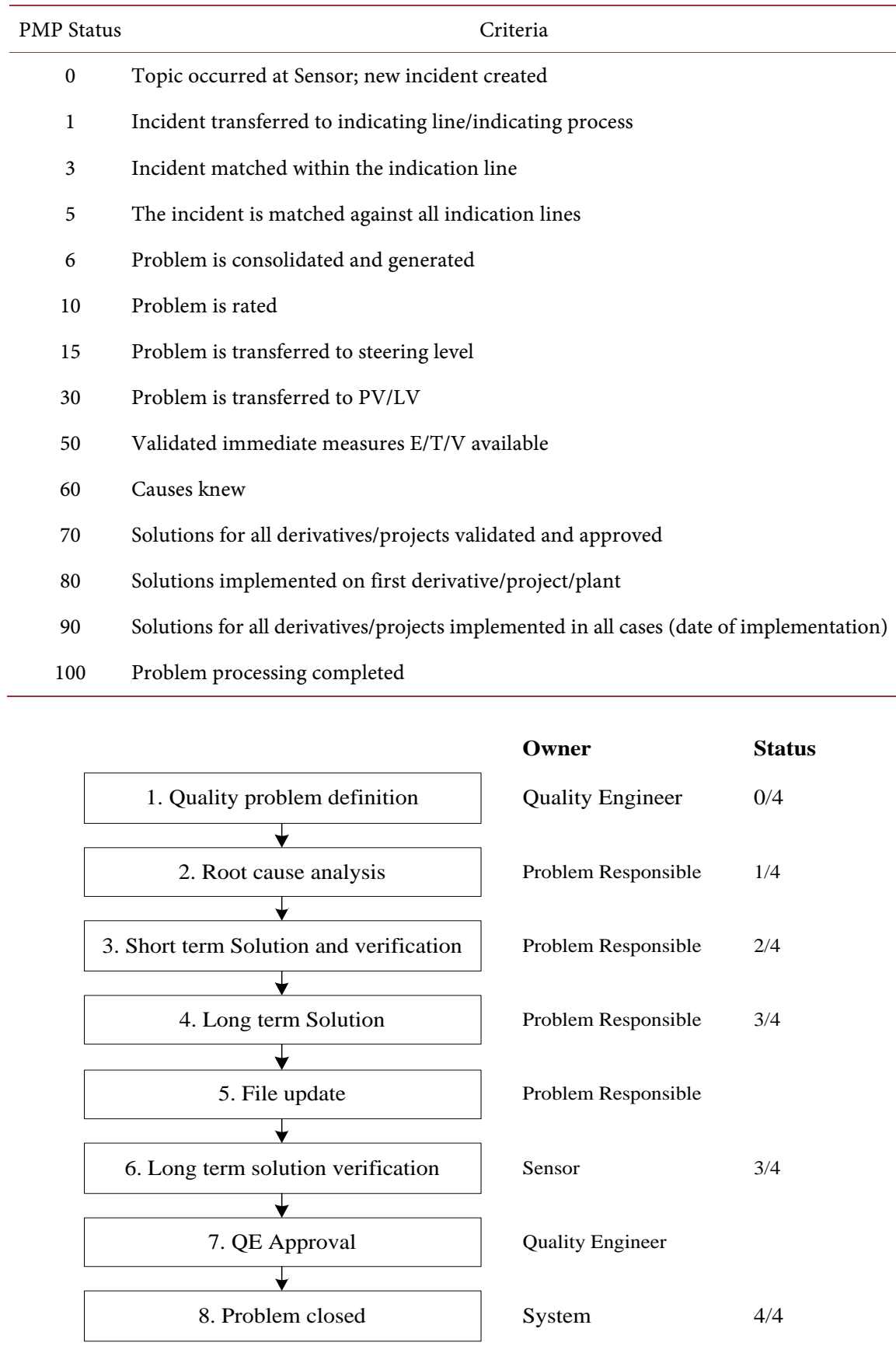

Figure 2. The problem-solving process at SGM.

BBA problem-solving process is more complicated and contains more problem status than SGM process. SGM divides problem-solving into four phases, which is relatively more concise. What's more, we found that the sequence of immediate measures and root cause analysis is slightly different. BBA first carries out immediate measures, then conduct root cause identification. SGM, on the other hand, performs the root cause analysis first and then takes short-term solutions. 
To uncover the factors that affecting problem-solving from a micro level, based on the five-stage model of problem solving (Xu et al., 2018), we analyse the following aspects of the problem-solving process, including, problem creation, problem definition, root cause analysis, solution formulation and implementation.

1) Problem creation

In $\mathrm{BBA}$, the sensors who detect the quality defects create PQM based on the $\mathrm{PQM}$ creation rules. The PQM creation rule describes when a PQM needs to be created. For example, all the problems discovered by the road test need to create $\mathrm{PQM}$. However, the rules are not very transparent, and sometimes they do not follow these rules. A worse situation is that they are not willing to expose the problem and do not create a PQM, which leads to the hidden of some problems. The advantage of this strategy is that the sensors have first-hand information on the problem and can input the relevant information of the problem into the PQM system with relatively high accuracy. However, problem creation is hard to be standardised due to the distribution of sensors.

In SGM, sensors do not create PCR; they input the quality problems into the Global Quality Data System (GSIP) system. Quality engineers daily review the data in this system and create PCR according to the PCR creation rules. They are well-trained and able to describe problems in a standardised way. However, they are not the sensors and don't have the first-hand information about the problem.

2) Problem definition: Striving for a common language.

One prerequisite for problem definition is developing a common descriptive language that can be understood across the department and organisational lines. Different people in different department use different words for the same problem. For example, the record "Fuel tank cover part not to specification" in GSIP system, is the same problem with the record "offset between fuel tank cover and body in white" in Audit III system in SGM. However, it is described in different languages. In another example, during the falling of the glass, some noise is sharp and harsh, and some noise is jittery noise, and the Sensor often records them as Disturbing noise.

To address this issue, BBA developed a set of standardised descriptions of quality problems, internally referred to as Vehicle Fault Coding (VFC). The VFC provides a set of standardised terms containing defect location, defect type, and defect condition. In the various quality management systems of BBA, the function of VFC has been introduced.

3) Root Cause Analysis (RCA)

RCA is a very important part of the problem-solving process. We found that Fishbone diagram analysis and "five whys" are commonly used in two case plants in RCA. When conducting fishbone diagram analysis, problem-solving team brainstorms together to analyse the most likely underlying root causes. We think it depends on personal experience heavily. At the same time, the "root" cause is often ignored. To solve problems faster, problem solvers formulate solutions for the shallow causes. Meanwhile, the "root" cause is still there which 
leads to the situation that some same type problems occur repeatedly. What's more, for some complex problems, it's difficult to discover all the root causes. So the contributing factors to the problem cannot be solved completely. An interesting phenomenon in two case plants is people seem to easily blame "operator". When we look through the root cause records, "operator mistake" occupy a large proportion. However, the other contributing factors such as material quality, material size, and equipment wear are not focused.

When conducting RCA, the root causes that were verified correctly are recorded in the PQM or PCR system. However, those non-real root causes are often not recorded, which we think are essential treasures to problem-solving because they will remind the problem solver not to take a detour.

4) Long term solution, not immediate measure

Immediate measures are taken to "fix" the problem and ensure the vehicles are of good quality before they are sent to the customers. While sometimes they are regarded as long-term solutions in production line especially in cases that root causes are difficult to uncover. After the vehicle is used for some time by the customer, the immediate measure will expire, and the problems come back. We saw some cases in BBA such as door noise problem, which is caused by the friction between the two mechanical devices inside the door panel. The immediate measure is to apply some grease to the mechanical devices before assembly. However, after the car ran for thousands of kilometres, the grease dried or stained with dust and the noise reappeared.

Sometimes the description of long term solutions is too general. When we look through the solution report in PCR at SGM plant, "strengthening inspections" and "strengthening the focus on issues" are some common long-term solutions, which is absolutely not the real long-term solution. For example, in PCR Number 152398628, the long-term solution description is: Operator will pay more attention to check the impurity and keep packaging material for steering tool clean. In this kind of situation, we think the long term solution description should be more precise and detailed.

\subsection{Problem-Solving Team}

1) Support but not lead

A complex problem-solving team usually contains employees from different departments, such as the quality department, four workshops, supplier quality, logistics, and so on. Because the cause of the problem is not clear, few departments in the team are willing to lead the problem-solving. The word we hear most in the two case factories is "support." Unclear team leader leads to the problem-solving meeting and activities inefficient. At BBA, this situation will be better. Because the analysis centre in BBA acts as a judge; their pre-analysis results tend to point to the main contributor who is often the leader of the problem-solving team.

2) To evade responsibility, rather than proactive to help

For some problems caused by many factors, it is difficult to find the responsi- 
ble party. Sometimes even if the problem-solving team outcrop potential contributors, the problem is trying to hand over. These potential contributors tend to prove that these problems are not caused by them, rather than think about what they can do to promote problem-solving.

3) Who knows how to solve?

For some complex problems, ordinary team members cannot propose solutions. At this point, the problem solving team needs to seek the help of experts. Sometimes the problem-solving member knows who the expert on the problem based on experience is, or who has previously solved a similar problem. However, in some cases, problem solving team members do not know who the expert in this area is.

We found an interesting case at BBA, which is E84 rear taillight cracking problem. The size of the taillight is not qualified or the dimensions of the body have deviation could be the potential root causes. Therefore, a measurement data is needed to prove who is contributing to this problem. However, the measurement benchmarks of the analysis center and body shop are inconsistent. Each side has their own modeling standards. Just when the two sides are deadlocked on the measurement benchmark for this problem, a senior manager from assembly shop who has been working in the analysis center for a long time, suggested to the analysis center how to find the benchmark and how to model it. The analysis center improved the measurement method and the measurement benchmark according to the manager's suggestion, and then unified the data with the car body shop. Finally, based on unified data, the problem solving team found the root cause. In this case, the senior manager was not a member of this problem team, but he had experience in solving related problems so he could propose a good solution. But on the other hand, if the manager does not take the initiative to make these suggestions, the problem solving team does not know that he is an expert in this area. This shows that know who is very important in quality problem solving.

4) Problem-solving team member not qualified

When we walked around the two plants, we found that many workers and even managers are very young, which is very different from some European vehicle manufactures. Due to the rapid development of China's auto industry, staff mobility is relatively large, which results in unqualified quality management employees. This situation makes it more difficult to solve the quality problem and reduce the problem solving efficiency. At the same time, higher requirements are placed on problem solving relying on data rather than experience.

\subsection{Accumulation and Use of Data, Information, and Knowledge}

Each step in the problem-solving process requires human knowledge and experience, but also creates new experience and knowledge. However, judging from the case companies, they not only lack effective management of knowledge but also lack knowledge acquisition and accumulation, which makes the "knowledge," a precious resource for quality management, wasted. Whether it is the 
Just Do It (JDT) related experience from the production line or the information and data recorded in the process of complex problem-solving. Whether it is stored in the system and Email, or the employee's memory, these experiences, data, and information are essential for problem-solving. Therefore, how to organise these experiences, data, and information, transform them into knowledge, and use the knowledge in the new problem-solving is an important issue worthy of study.

Below we analyse the accumulation and use of data, information, and knowledge of the case plants in the process of problem-solving.

1) Lack of long-term data analysis

In the survey, we found that the case plants tend to focus on issues that are happening right now, and they rarely carry out systematic analysis and summary of long-standing quality problems. In this situation, problem-solving turns to be a "firefighting" behaviour. The focus of quality management personnel often shifts with the emergence of new quality issues. If every problem is treated equally, it will waste many workforces and material resources. Grasping the fundamental problems, prioritising the problems, and looking at the problems from a global perspective rather than a local perspective, will effectively use problem-solving resources to reduce the cost of human and material resources for the company.

2) Poor data collection and storage

The quality problem solving team rarely uses some quality tools to collect information about defects. At the same time, there is a lack of unified archiving of data during problem-solving. The only part of the data is stored in PQM or PCR. However, much data is transmitted via email, telephone, or stored in a free disk. Document and data stored in two case companies' quality management information systems are incomplete.

3) Lack of utilisation of data, information, and knowledge

Both the BBA's PQM system and the SGM's PCR system store a large number of historical data. However, we found that they often do not attract people's attention. When a new problem occurs, employees tend to use memory to judge whether the problem has occurred or not, or inquire the relevant person by mail or phone, instead of going to the systems to check the historical record. At the same time, due to the limitation of the search function in PQM and PCR system, problem solvers do not have easy access to the root cause and solution knowledge.

\section{Questionnaire Survey Results}

Questionnaire survey results show that the questions raised in Section 3 are also reflected in the questionnaire. We calculated the scores of each question in the questionnaire and tried to find out the critical factors that affected the problem-solving. We extracted those questions with an average score below 4 points and renumbered them to form the results shown in Table 4. In the five-rank Likert 
Table 4. Low satisfactory questions at two case companies.

\begin{tabular}{|c|c|c|c|}
\hline \multirow{2}{*}{ No. } & \multirow{2}{*}{ Item } & \multicolumn{2}{|c|}{ Average Score } \\
\hline & & BBA & SGM \\
\hline 1 & Quality problem definition is clear and with a unified standard & 3.4 & 3.8 \\
\hline 2 & Quality-related data are recorded clearly and accurately. & 3.8 & 3.6 \\
\hline 3 & The quality analysis equipment is ample and advanced & 4.6 & 3.8 \\
\hline 4 & $\begin{array}{l}\text { The results of the analysis can always be recognised by quality } \\
\text { executives and have a strong convincing }\end{array}$ & 3.7 & 3.2 \\
\hline 5 & Lesson Learned can be well stored and documented & 3.8 & 3.8 \\
\hline 6 & The problem-solving team leader is clear & 2.8 & 3.4 \\
\hline 7 & $\begin{array}{l}\text { The problem-solving team collects and uses a wide range of } \\
\text { data, information and knowledge to solve the problem. }\end{array}$ & 3.5 & 3.3 \\
\hline 8 & Data, information, and knowledge, can be found promptly & 3.3 & 3.1 \\
\hline
\end{tabular}

scale, one represents strong disagree, two represents disagree, three represents neither disagree nor agree, four represents agree, and five represents strongly agree.

From Table 4 we can see that the employees of the two companies are between either disagree nor agree and agree concerning question $1,2,4,5,6,7$, and 8 . The result shows that quality management stakeholders believe that the company needs improvement in these areas. For the third question, BBA scored higher than SGM, which means that BBA's analysis equipment is more abundant than SGM, and quality management personnel are also satisfied. Overall, the results of the questionnaire and the survey results in Section 3 are more consistent. In the next section, we will propose a comprehensive solution to these problems, especially the use of data, information, and knowledge in quality management.

\section{Proposals to Improve Problem-Solving}

\subsection{Knowledge Management Based Intelligent Problem-Solving System (IPSS)}

Through investigation, it is found that the lack of utilisation of data, information, and knowledge of quality management, especially in problem-solving, is a common problem in typical automobile companies. In the era of informationization where the level of automation is getting higher and higher, and the level of digitisation is gradually increasing, the manufacturing industry has accumulated a great deal of data in the process of production and quality management. How to make these data fully play a role in quality management is a question we raise. What's more, some automotive companies like BBA and SGM, which are young and growing organisations, are also facing the problem of employee turnover which leads to the turnover of experience. Under this circumstance, there is an increasing demand for these companies to accumulate its problem-solving data and make full use of this data for better decision making in 
quality management which finally will increase the product quality. Based on this idea, we propose knowledge management based intelligent problem-solving system (IPSS). At the same time, we regard the five aspects of technological environment construction (TEC), personnel quality construction (PQC), organisational environment construction (OEC), institutional environment construction (IEC) and cultural environment construction (CEC) as the guarantee of this model.

The structure of the IPSS is shown in Figure 3.

Based on the problem-solving process, as well as internal and external knowledge resources, the architecture shown in Figure 3 can be summarised as "4321 architecture", which is "four subsystems, three databases, two levels, one goal." The architecture expresses the whole process from data collection to data processing and then using problem-solving knowledge to support problem-solving.

The four subsystems refer to Data Acquisition System (DAS), Ontology Generating Module (OGM), Knowledge Transformation Module (KTM) and Knowledge Inference Module (KIM). The three databases refer to the Problem-Solving Database (PSDB), the standard terminology and ontology database and Problem-Solving Knowledge Base (PSKB). The two levels refer to the application system level and the primary system level, which are: front-end data acquisition system and decision support system; background knowledge processing system and background maintenance system. A goal refers to all the data, information and knowledge in the system serve the goal of solving quality problems.

DAS is used for collecting various data during problem-solving. For example, IPS-Q, GSIP, PQM, and PCR in the two case companies can all be regarded as data acquisition systems. Based on various data in DAS, OGM builds a standardised and unified ontology of problems, immediate measures, root causes and long-term solutions. These ontologies serve as the universal language for communication among various departments, and also lay the foundation for the

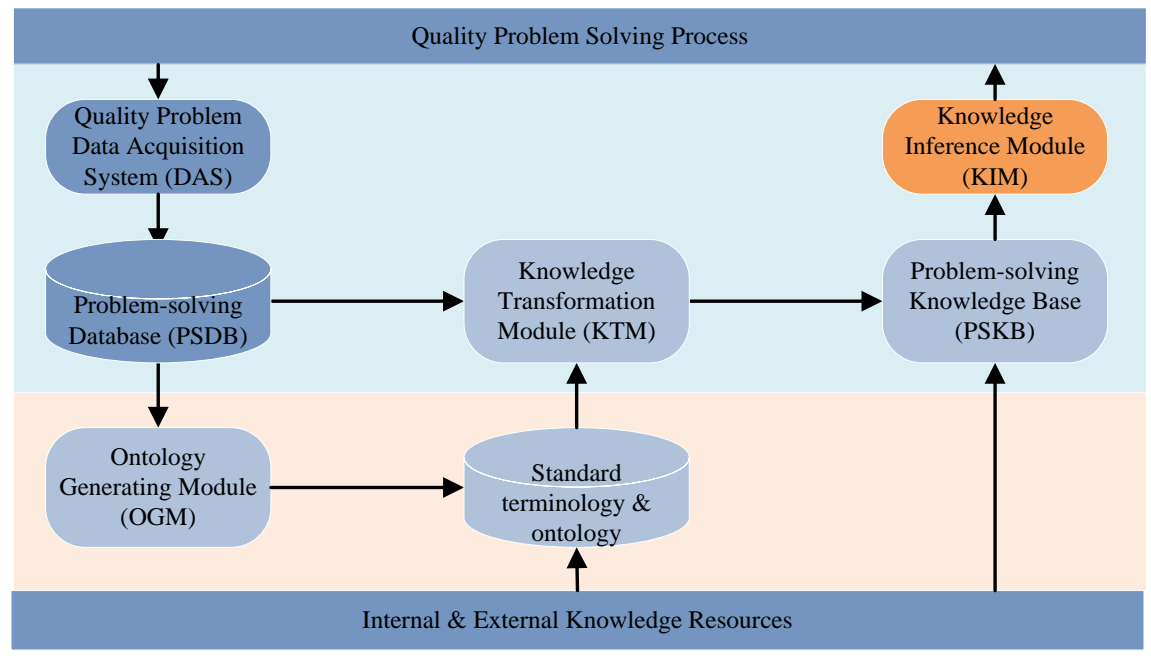

Figure 3. The architecture of knowledge management based intelligent problem-solving system (IPSS). 
construction of subsequent knowledge bases. Based on the ontology constructed above, the KTM uses a series of intelligent algorithms to transform the original data into corresponding knowledge which constitute the PSKB, including quality problem knowledge base, immediate measure knowledge base, root cause knowledge base and solution knowledge base. These knowledge bases serve as the basis for the inference of new quality problems and are the core part of the intelligent problem-solving system.

The KIM provides various knowledges to the user when the user retrieves the knowledge base system. Knowledge inference process is shown in Figure 4.

Step 1. The user inputs the problems with the free text, the system pre-processes the query and calculates the similarity between the text of the query and the quality problem knowledge base. Then the system extracts the quality problem with similarity higher than a certain threshold to form a set of quality problems.

Step 2. When the user clicks on the immediate measure in the system interface, the system outputs all the sets of historical immediate measures for the problem based on the extracted problem sets and the entity relationship diagram (ERD) between the quality problem knowledge base and the Immediate Measure knowledge base.

Step 3. When the user clicks on the root cause in the system interface, based on the extracted problem set, and the ERD of the quality problem knowledge base and the root cause knowledge base, the system outputs and presents the root causes set in the form of a fishbone diagram.

Step 4. When the user clicks on the solution in the system interface, based on the output root cause, and the ERD between the root cause knowledge base and the solution knowledge base, the system outputs the solution set for this root cause.

IPSS provides problem solvers with appropriate knowledge at different stages of quality problem analysis and resolution. This knowledge includes what immediate measures are taken for each problem, what are the root causes of the

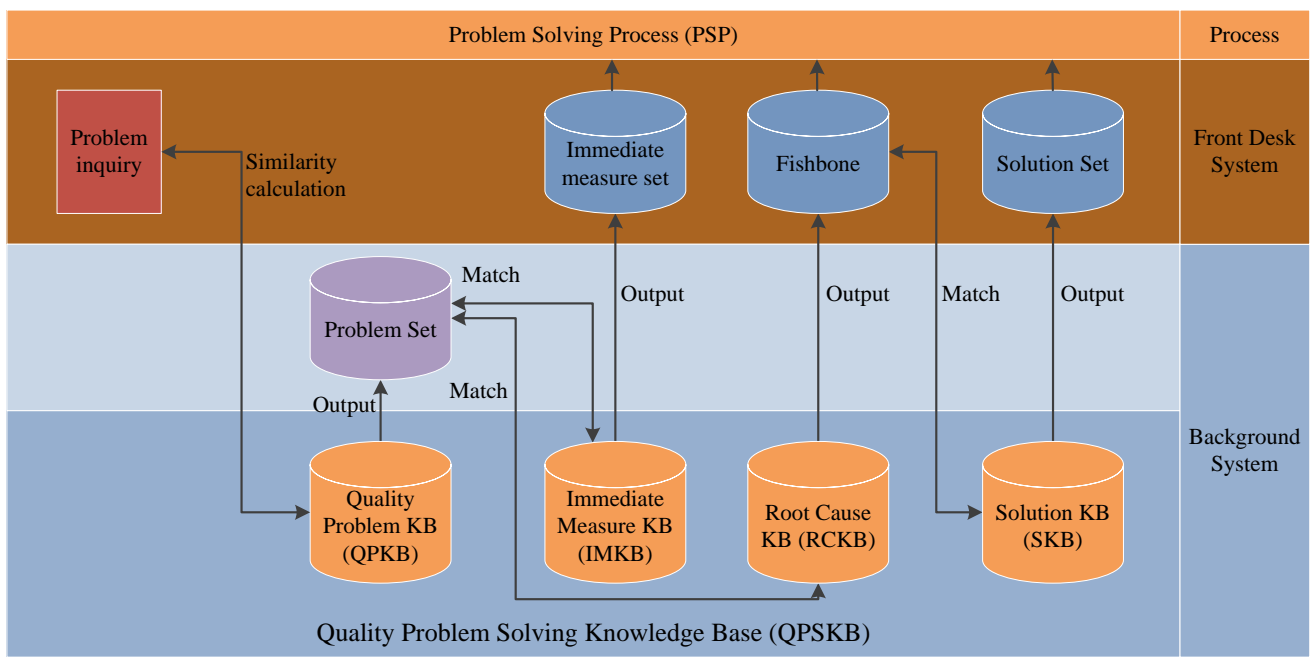

Figure 4. Knowledge inference process. 
problem, and what are the long-term solutions to the problem? Not only that, the system can provide "know-who" at all stages of problem-solving, providing relational knowledge for domain experts. This system, similar to a question-and-answer engine, improves the efficiency and effectiveness and reduces the cost of problem-solving. At the same time, from the perspective of company $\mathrm{KM}$, the system provides a systematic method of knowledge storage, accumulation, transformation, and application.

\subsection{A Five-Tier Environment to Ensure the Implementation of IPSS}

In addition to the architecture of Figure 3, IPSS needs five aspects to ensure its successful implementation, including technological environment construction (TEC), personnel quality construction (PQC), organisational environment construction (OEC), institutional environment construction (IEC) and cultural environment construction (CEC). These five elements are shown in Figure 5.

The TEC is mainly to build an information network environment for the overall architecture of KM in Figure 3. TEC uses information technology, database technology, and network communication technology to develop intelligent problem-solving technology systems. The PQC means that all employees need to have specific KM literacy. Quality management and problem-solving are related to every employee in the company, so is KM. Experience and knowledge exist in the brains of employees consist of their wealth. Whether employees are willing to share their own experience with others is a fundamental issue in KM. If employees are not willing to share, the best methods and techniques are useless. Because of the hidden and unspoken nature of knowledge, even if employees are willing to share, it will be helpless due to the lack of feasible expressions. So it requires training to improve the quality and expression ability of employees. OEC refers to how to coordinate numerous related personnel and employees involved in problem-solving and form a specific organisational model. From the perspective of IEC, the company needs to formulate relevant incentive systems and form incentive mechanisms for KM so that every employee should regard $\mathrm{KM}$ as "work within the company" instead of "extra burden." As for CEC, it

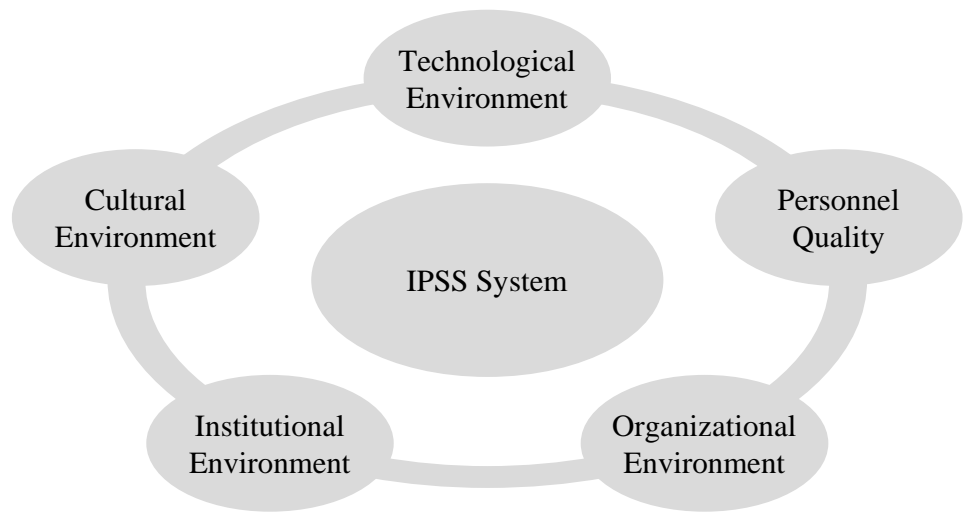

Figure 5. Construction of " $1+5$ " for IPSS. 
takes a relatively long period of mechanical cultivation. If the $\mathrm{KM}$ system is a "hard constraint," then the KM culture is a "soft constraint."

IPSS is a development strategy of the company and should be incorporated into the overall development strategy of the company. KM work is also a strategic job. Whether the accumulation of knowledge or the formation of collective wisdom is a process, its effect will not be immediate, and its value is also strategic. KM work is generally considered to be "important but not urgent" and is often squeezed by other "urgent" things, which makes it important for companies to have "strategic strength" and "strategic patience."

\section{Conclusion}

With a case study of two automotive manufacturers, this research investigates the problem-solving of two typical automotive plants to explore the key factors affecting problem-solving. Two plants which are owned by joint ventures in China are selected for the study. By comparing and analysing the status quo and problems in problem-solving of these two plants from the perspective of KM via interviews, questionnaire distribution and filed work activities in the case plants, we detected the key factors that influence problem-solving. The result shows that there is a lack of utilisation of data, information, and knowledge in problem-solving in the automotive industry.

According to the analysis results, we propose knowledge management based intelligent problem-solving system (IPSS). By the current problem-solving process and utilising basic knowledge inside and outside the company, IPSS utilises the historical problem-solving data to generate a common language for the description of problems, immediate measure, root causes and long-term solutions. Then it transfers the historical problem-solving data into useful knowledge which in turn support the new quality problem-solving via the system interface. A five-tier environment is also proposed to ensure the successful implementation of IPSS.

The method and the system structure make the quality management process, especially problem-solving step by step towards the process of knowledge and wisdom, and gradually increase the efficiency and effectiveness of problem-solving.

\section{Acknowledgements}

The authors would like to thank the Institute of Systems Engineering of the Dalian University of Technology and BMW Brilliance Automotive Ltd. for supporting the research. This work was also supported by the National Natural Science Foundation of China (NSFC) Grant [72001034, 71871041].

\section{Conflicts of Interest}

The authors declare no conflicts of interest regarding the publication of this paper.

\section{References}

Asan, U., \& Soyer, A. (2016). Failure Mode and Effects Analysis under Uncertainty: A Li- 
terature Review and Tutorial. In C. Kahraman, \& S. Yanik (Eds), Intelligent Decision Making in Quality Management: Theory and Applications (pp. 265-325). Springer International Publishing.

Aslan, B., Ekinci, Y., \& Toy, A. Ö. (2016). Special Control Charts Using Intelligent Techniques: EWMA Control Charts. In C. Kahraman, \& S. Yanik (Eds), Intelligent Decision Making in Quality Management: Theory and Applications (pp. 101-125). Springer International Publishing.

Berends, H. (2005). Exploring Knowledge Sharing: Moves, Problem Solving and Justification. Knowledge Management Research \& Practice, 3, 97-105.

Ch, F. A., Khobreh, M., Nasiri, S., \& Fathi, M. (2009). Knowledge Management Support for Quality Management to Achieve Higher Customer Satisfaction. Processing of 2009 IEEE International Conference on Electro/Information Technology, Windsor, 7-9 June 2009, 78-83.

Chen, F., Deng, P., Wan, J., Zhang, D., Vasilakos, A. V., \& Rong, X. (2015). Data Mining for the Internet of Things: Literature Review and Challenges. International Journal of Distributed Sensor Networks, 2015, Article ID: 431047.

https://doi.org/10.1155/2015/431047

Choo, A. S., Linderman, K. W., \& Schroeder, R. G. (2007). Method and Context Perspectives on Learning and Knowledge Creation in Quality Management. Journal of Operations Management, 25, 918-931. https://doi.org/10.1016/j.jom.2006.08.002

Erginel, N., \& Şentürk, S. (2015). Intelligent Systems in Total Quality Management. Intelligent Systems Reference Library, 87, 407-430.

https://doi.org/10.1007/978-3-319-17906-3 16

Fine, C. H. (1986). Quality Improvement and Learning in Productive Systems. Management Science, 32, 1301-1315. https://doi.org/10.1287/mnsc.32.10.1301

Hanaysha, J., Hilman, H., \& Abdul-Ghani N. H. (2014). Direct and Indirect Effects of Product Innovation and Product Quality on Brand Image: Empirical Evidence Automotive Industry. FEBS Letters, 388, 213-225.

Herrmann, A., Henneberg, S. C., \& Landwehr, J. (2010). Squaring Customer Demands, Brand Strength, and Production Requirements: A Case Example of an Integrated Product and Branding Strategy. Total Quality Management \& Business Excellence, 21, 1017-1031. https://doi.org/10.1080/14783363.2010.487706

Ibrahim, S. B., \& Heng, L. H. (2013). Learning and Knowledge Management: Learning as an Integrative Role for Knowledge Creation. International Conference on Informatics and Creative Multimedia, Kuala Lumpur, 4-6 September 2013, 209-214.

Lari, A. (2004). A Decision Support System for Solving Quality Problems Using Case-Based Reasoning. Total Quality Management \& Business Excellence, 14, 733-745.

Liang, K., \& Zhang, Q. (2010). Study on the Organizational Structured Problem Solving on Total Quality Management. International Journal of Business \& Management, 5, 178-183. https://doi.org/10.5539/ijbm.v5n10p178

Linderman, K., Schroeder, R. G., Zaheer, S., Liedtke, C., \& Choo, A. S. (2004). Integrating Quality Management Practices with Knowledge Creation Processes. Journal of Operations Management, 22, 589-607. https://doi.org/10.1016/j.jom.2004.07.001

Liu, H. C., Liu, L., \& Li, P. (2014). Failure Mode and Effects Analysis Using Intuitionistic Fuzzy Hybrid Weighted Euclidean Distance Operator. International Journal of Systems Science, 45, 1-19. https://doi.org/10.1080/00207721.2012.760669

Liu, H. C., Liu, L., \& Lin, Q. L. (2013a). Fuzzy Failure Mode and Effects Analysis Using Fuzzy Evidential Reasoning and Belief Rule-Based Methodology. IEEE Transactions on 
Reliability, 62, 23-36.

Liu, H. C., Liu, L., \& Liu, N. (2013b). Risk Evaluation Approaches in Failure Mode and Effects Analysis: A Literature Review. Expert Systems with Applications, 40, 828-838.

Liu, H. C., Liu, L., Liu, N., \& Mao, L.-X. (2012). Risk Evaluation in Failure Mode and Effects Analysis with Extended VIKOR Method under Fuzzy Environment. Expert Systems with Applications, 39, 12926-12934. https://doi.org/10.1016/j.eswa.2012.05.031

Macduffie, J. P. (1997). The Road to "Root Cause": Shop-Floor Problem-Solving at Three Auto Assembly Plants. Management Science, 43, 479-502.

https://doi.org/10.1287/mnsc.43.4.479

Mons, B., Van, H. H., Chichester, C., Hoen, P. B., den Dunnen, J. T., van, O. G., \& Schultes, E. (2011). The Value of Data. Nature Genetics, 43, 281-283. https://doi.org/10.1038/ng0411-281

Peachey, T. A., \& Hall, D. J. (2006). Supporting Complex Problems: An Examination of Churchman's Inquirers as a Knowledge Management Foundation. Knowledge Management Research \& Practice, 4, 197-206. https://doi.org/10.1057/palgrave.kmrp.8500100

Postrel, S. (2002). Islands of Shared Knowledge: Specialization and Mutual Understanding in Problem-Solving Teams. Organization Science, 13, 303-320. https://doi.org/10.1287/orsc.13.3.303.2773

Ruikar, K., Anumba, C. J., \& Egbu, C. (2007). Integrated Use of Technologies and Techniques for Construction Knowledge Management. Knowledge Management Research \& Practice, 5, 297-311.

Srikanth, K., Harish, A., Heymaraju, C. H., \& Ashok Kumar, N. (2010). Intelligent Quality Management Expert System Using PA-AKD in large Databases. International Journal of Engineering Science and Technology, 2, 632-636.

Stanleigh, M. (2013). Future Trends in Quality Management. Project Management Articles for Project Managers.

https://pmhut.com/future-trends-in-quality-management

Wang, X. (2009). Intelligent Quality Management Using Knowledge Discovery in Databases. International Conference on Computational Intelligence and Software Engineering, Wuhan, 11-13 December 2009, 1-4.

Xu, Z., \& Dang, Y. (2020). Automated Digital Cause-and-Effect Diagrams to Assist Causal Analysis in Problem-Solving: A Data-Driven Approach. International Journal of Production Research, 58, 5359-5379. https://doi.org/10.1080/00207543.2020.1727043

Xu, Z., Dang, Y., \& Munro, P. (2018). Knowledge-Driven Intelligent Quality Problem Solving System in the Automotive Industry. Advanced Engineering Informatics, 38, 441-457. https://doi.org/10.1016/j.aei.2018.08.013

Xu, Z., Dang, Y., Munro, P., \& Wang, Y. (2020). A Data-Driven Approach for Constructing the Component-Failure Mode Matrix for FMEA. Journal of Intelligent Manufacturing, 31, 249-265. https://doi.org/10.1007/s10845-019-01466-Z 


\section{Appendix 1. Closed Questions in the Questionnaire}

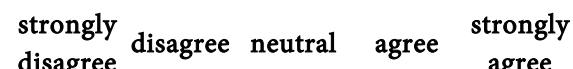

\section{Problem-solving process}

\subsection{Quality problem definition}

1) Quality problem definition is clear and with unified standard

2) The problem description is clear and can be understood by various departments

3) Quality-related data are recorded clear and accurate.

4) The evaluation criteria for the severity of the problem or defect are uniform and can be understood and applied by the staff of the relevant departments

$\begin{array}{lllll}1 & 2 & 3 & 4 & 5 \\ 1 & 2 & 3 & 4 & 5 \\ 1 & 2 & 3 & 4 & 5 \\ 1 & 2 & 3 & 4 & 5\end{array}$

\subsection{Problem Analysis}

1) The quality analysis equipment is ample and advanced

2) The quality problems can be analysed in time and the analysis results can be obtained in time

3) Analysis results are recognized by the problem-solving team and are very persuasive

4) Analysis of the results can always be recognized by quality executives and has a strong convincing

$\begin{array}{lllll}1 & 2 & 3 & 4 & 5 \\ 1 & 2 & 3 & 4 & 5 \\ 1 & 2 & 3 & 4 & 5 \\ 1 & 2 & 3 & 4 & 5\end{array}$

\subsection{Root cause analysis (RCA)}

1) Carry out RCA scientifically rather than directly to the proposed solution

2) RCA relies mainly on personal experience rather than data

3) RCA tools is diversified and can be understood and used by most employees

4) The root cause of the problem can always be found and comprehensive

\subsection{Solution}

1) Solution implementation and validation has certain standards

2) Temporary solutions are used as long term solution

3) The solutions will be evaluated and then implemented

4) Implementation effect of the solution will been evaluated

\subsection{Lessons Learned (LeLe)}

1) The company do lesson learn after the problem is solved

2) LeLe can be well stored and documented

3) LeLe plays an important role in solving new quality problems

\section{Human factors}

\subsection{Leadership}

1) The CEO/Administrator is a primary driving force behind quality improvement efforts.

2) The senior executives allocate adequate organizational resources to improving quality.

3) The senior executives have articulated a clear vision for improving the quality of products.

4) The senior executives act on suggestions to improve the quality of products.

\section{2. problem-solving team}

1) When quality problems occur, can quickly organize the relevant personnel to form the problem-solving team

$\begin{array}{lllll}1 & 2 & 3 & 4 & 5 \\ 1 & 2 & 3 & 4 & 5 \\ 1 & 2 & 3 & 4 & 5 \\ 1 & 2 & 3 & 4 & 5 \\ & & & & \\ 1 & 2 & 3 & 4 & 5\end{array}$




\section{Continued}

2) problem-solving team leader is clear

$\begin{array}{lllll}1 & 2 & 3 & 4 & 5\end{array}$

3) The problem-solving team effectively coordinate their efforts with others to solve the quality problems

4) The team members have a certain level of competency and problem-solving skills

1

$2 \quad 3 \quad 4 \quad 5$

3. Data, Information \& Knowledge

1) Problem-solving team collects and uses a wide range of data, information and knowledge to solve the problem.

2) The company continually tries to improve how it uses data, information and knowledge on the problem-solving.

3) Quality data can be recorded and documented with accuracy

4) Data, information and knowledge can be found in a timely manner

1

$\begin{array}{llll}2 & 3 & 4 & 5\end{array}$

5) Data, information and knowledge can be effectively shared between the relevant departments

\begin{tabular}{lllll}
1 & 2 & 3 & 4 & 5 \\
1 & 2 & 3 & 4 & 5 \\
1 & 2 & 3 & 4 & 5 \\
1 & 2 & 3 & 4 & 5 \\
1 & 2 & 3 & 4 & 5 \\
\hline
\end{tabular}

